https://doi.org/10.48048/tis.2022.2070

\title{
On Generalized Mock Theta Functions of Tenth Order
}

\section{Pramod Kumar Rawat}

Department of Mathematics and Astronomy, University of Lucknow, Lucknow 226007, India

(Corresponding author's e-mail: pramodkrawat@yahoo.com)

Received: 8 September 2020, Revised: 25 May 2021, Accepted: 8 June 2021

\begin{abstract}
In this paper we have given 3 independent variable generalization of tenth order mock theta functions and have found some relations between generalized mock theta functions of different order. We have given a continued fraction representation of generalized tenth order mock theta functions. We have also given the q-integral representation and multibasic expansion for these functions. Further by specializing the parameters we have connected mock theta functions with continued fraction of Ramanujan.
\end{abstract}

Keywords: Mock theta functions, q-hypergeometric series, Continued fraction, q-integrals, Multibasic expansion

\section{Introduction}

Ramanujan's first letter to Hardy contains several results on continued fraction;

$\mathrm{R}(\mathrm{q})=\frac{1}{1+1+1+1+\cdots} \frac{q}{q^{2}} \frac{q^{3}}{1+}$

After the discovery of Ramanujan's lost note book Andrews proved these results. On page 365 of his "lost" note book Ramanujan wrote 5 modular equations related $R(-q), R\left(q^{2}\right), R\left(q^{3}\right), R\left(q^{4}\right)$ and $R\left(q^{5}\right)$. Later Denis [12-14]; Bhargava [4,5] and many more proved many results related to $R(q)$.

From his death bed Ramanujan again wrote a letter to Hardy [18] giving 17 functions and called them mock theta functions, 4 of them of third order, 10 of fifth order and 3 of seventh order. Though Ramanujan did not clarify what he meant by "order" of the mock theta functions. Later 4 more mock theta functions were discovered by Choi [6] from Ramanujan's "Lost" notebook. Choi studied these tenth order mock theta functions in detail in papers [7-9]. Srivastava [20,21] also studied these tenth order mock theta functions and expressed them as a continued fraction. Zwegers [23] proved the identities of tenth order mock theta function with different approach.

Choi [10] generalized tenth order mock theta function and found relations between them. Andrews and Yee [2] gave the relations between 2 mock theta functions $\omega(q)$ and $v(q)$. Recently Berndt et al. [3] generalized the identities and found more results.

In this paper, we have generalized these tenth-order mock theta functions by introducing 3 independent variables. The advantage of introducing 3 variables is that we have 3 extra parameters and, by specializing them, we get some known functions and some new identities. We list them in the last section and also give relations between generalized mock theta functions of different orders. The tenth order mock theta functions of Ramanujan are;

$\Phi(q)=\sum_{n=0}^{\infty} \frac{q^{n(n+1) / 2}}{\left(q ; q^{2}\right)_{n+1}}$,
$\Psi(q)=\sum_{n=0}^{\infty} \frac{q^{(n+1)(n+2) / 2}}{\left(q ; q^{2}\right)_{n+1}}$, 
$\mathrm{X}(q)=\sum_{n=0}^{\infty} \frac{(-1)^{n} q^{n^{2}}}{(-q ; q)_{2 n}}$

$\chi(q)=\sum_{n=0}^{\infty} \frac{(-1)^{n} q^{(n+1)^{2}}}{(-q ; q)_{2 n+1}}$

where, $\left(a ; q^{k}\right)_{n}=\prod_{j=0}^{n-1}\left(1-a q^{k j}\right),\left(a ; q^{k}\right)_{0}=1,\left(a ; q^{k}\right)_{\infty}=\prod_{j=0}^{\infty}\left(1-a q^{k j}\right)$.

\section{Generalized tenth order mock theta functions}

We give a generalization of these tenth order mock theta functions by introducing 3 independent variables;

$\Phi(t, \alpha, z ; q)=\frac{1}{(t)_{\infty}} \sum_{n=0}^{\infty} \frac{(t)_{n} q^{n(n+1) / 2} z^{2 n} \alpha^{n}}{\left(z q ; q^{2}\right)_{n+1}}$,

$\Psi(t, \alpha, z ; q)=\frac{1}{(t)_{\infty}} \sum_{n=0}^{\infty} \frac{(t)_{n} q^{(n+1)(n+2) / 2} z^{2 n} \alpha^{n}}{\left(z q ; q^{2}\right)_{n+1}}$

$\mathrm{X}(t, \alpha, z ; q)=\frac{1}{(t)_{\infty}} \sum_{n=0}^{\infty} \frac{(t)_{n}(-1)^{n} q^{n^{2}} z^{2 n} \alpha^{n}}{(-z q ; q)_{2 n}}$

$\chi(t, \alpha, z ; q)=\frac{1}{(t)_{\infty}} \sum_{n=0}^{\infty} \frac{(t)_{n}(-1)^{n} q^{(n+1)^{2}} z^{2 n} \alpha^{n}}{(-z q ; q)_{2 n+1}}$

for $t=0, \alpha=1$, and $z=1$ they reduce to Ramanujan's tenth order mock theta functions.

\section{Differential equations}

The differential operator $D_{q}$ is defined as [9];

$z D_{q, z} F(z, \alpha)=F(z, \alpha)-F(z q, \alpha)$.

Following differential equations are true for these functions.

(i) $D_{q, t}^{m} \Phi(t, \alpha, z ; q)=\Phi\left(t, \alpha q^{m}, z ; q\right)$,

(ii) $D_{q, t}^{m} \Psi(t, \alpha, z ; q)=\Psi\left(t, \alpha q^{m}, z ; q\right)$,

(iii) $D_{q, t}^{m} \mathrm{X}(t, \alpha, z ; q)=\mathrm{X}\left(t, \alpha q^{m}, z ; q\right)$,

(iv) $D_{q, t}^{m} \chi(t, \alpha, z ; q)=\chi\left(t, \alpha q^{m}, z ; q\right)$,

(v) $D_{q, t}^{m} \Phi(t, \alpha, z ; q)=\frac{1}{q} \Psi\left(t, \alpha q^{m}, z ; q\right)$,

(vi) $\mathrm{X}(t, \alpha, z ; q)=\frac{1}{(t)_{\infty}} \sum_{n=0}^{\infty} \frac{(t)_{n}(-1)^{n} q^{n^{2}} z^{2 n} \alpha^{n}}{(-z q ; q)_{2 n+1}}+z \chi(t, \alpha, z ; q)$. 


\section{Proof of (i)}

Applying the operator $D_{q, t}$ on $\Phi(t, \alpha, z ; q)$ we get

$$
\begin{aligned}
& t D_{q, t} \Phi(t, \alpha, z ; q)=\frac{1}{(t)_{\infty}} \sum_{n=0}^{\infty} \frac{(t)_{n} q^{n(n+1) / 2} z^{2 n} \alpha^{n}}{\left(z q ; q^{2}\right)_{n+1}}-\frac{1}{(t q)_{\infty}} \sum_{n=0}^{\infty} \frac{(t)_{n} q^{n(n+1) / 2} z^{2 n} \alpha^{n}}{\left(z q ; q^{2}\right)_{n+1}} \\
& =\frac{1}{(t)_{\infty}} \sum_{n=0}^{\infty} \frac{(t)_{n} q^{n(n+1) / 2} z^{2 n} \alpha^{n}}{\left(z q ; q^{2}\right)_{n+1}}-\frac{1}{(t)_{\infty}} \sum_{n=0}^{\infty} \frac{(t)_{n} q^{n(n+1) / 2} z^{2 n} \alpha^{n}}{\left(z q ; q^{2}\right)_{n+1}}\left(1-t q^{n}\right) \\
& =\frac{t}{(t)_{\infty}} \sum_{n=0}^{\infty} \frac{(t)_{n} q^{n(n+1) / 2} z^{2 n} \alpha^{n} q^{n}}{\left(z q ; q^{2}\right)_{n+1}} \\
& =t \Phi(t, \alpha q, z ; q)
\end{aligned}
$$

Applying the operator $m$ times, we get;

$D_{q, t}^{m} \Phi(t, \alpha, z ; q)=\Phi\left(t, \alpha q^{m}, z ; q\right)$

which proves (i). Similarly applying the operator $\mathrm{m}$ times on the other functions, we get (ii) - (iv).

\section{Proof of (v)}

From Eq. (2), for $\mathrm{m}=1$, we have

$$
\begin{aligned}
& D_{q, t} \Phi(t, \alpha, z ; q)=\Phi(t, \alpha q, z ; q) \\
& =\frac{1}{(t)_{\infty}} \sum_{n=0}^{\infty} \frac{(t)_{n} q^{n(n+1) / 2} z^{2 n} \alpha^{n} q^{n}}{\left(z q ; q^{2}\right)_{n+1}} \\
& =\frac{1}{q} \frac{1}{(t)_{\infty}} \sum_{n=0}^{\infty} \frac{(t)_{n} q^{(n+1)(n+2) / 2} z^{2 n} \alpha^{n} q^{n}}{\left(z q ; q^{2}\right)_{n+1}} \\
& =\frac{1}{q} \Psi\left(t, \alpha q^{m}, z ; q\right)
\end{aligned}
$$

\section{Proof of (vi)}

$$
\begin{aligned}
& \mathrm{X}(t, \alpha, z ; q)=\frac{1}{(t)_{\infty}} \sum_{n=0}^{\infty} \frac{(t)_{n}(-1)^{n} q^{n^{2}} z^{2 n} \alpha^{n}}{(-z q ; q)_{2 n}} \\
& =\frac{1}{(t)_{\infty}} \sum_{n=0}^{\infty} \frac{(t)_{n}(-1)^{n} q^{n^{2}} z^{2 n} \alpha^{n}\left(1+z q^{2 n+1}\right)}{(-z q ; q)_{2 n+1}} \\
& =\frac{1}{(t)_{\infty}} \sum_{n=0}^{\infty} \frac{(t)_{n}(-1)^{n} q^{n^{2}} z^{2 n} \alpha^{n}}{(-z q ; q)_{2 n+1}}+\frac{z}{(t)_{\infty}} \sum_{n=0}^{\infty} \frac{(t)_{n}(-1)^{n} q^{n^{2}+2 n+1} z^{2 n} \alpha^{n}}{(-z q ; q)_{2 n+1}} \\
& =\frac{1}{(t)_{\infty}} \sum_{n=0}^{\infty} \frac{(t)_{n}(-1)^{n} q^{n^{2}} z^{2 n} \alpha^{n}}{(-z q ; q)_{2 n+1}}+z \chi(t, \alpha, z ; q) .
\end{aligned}
$$




\section{Relations between generalized mock theta functions and partial mock theta functions of tenth order}

We prove the relations between generalized mock theta functions and generalized partial mock theta functions of tenth order.

To denote the partial sum of mock theta functions we write suffix $N$, so;

$\Phi_{N}(t, \alpha, z ; q)=\frac{1}{(t)_{\infty}} \sum_{n=0}^{N} \frac{(t)_{n} q^{n(n+1) / 2} z^{2 n} \alpha^{n}}{\left(z q ; q^{2}\right)_{n+1}}$

We will use the summation formula [16];

$$
\sum_{r=0}^{p} \alpha_{r} \beta_{r}=\beta_{p+1} \sum_{r=0}^{p} \alpha_{r}+\sum_{m=0}^{p}\left(\beta_{m}-\beta_{m+1}\right) \sum_{r=0}^{m} \alpha_{r}
$$

to prove the following relations between generalized mock theta functions.

(i) $\Psi_{p}(0, \alpha, z ; q)=q^{p+2} \Phi_{p}(0, \alpha, z ; q)+(1-q) \sum_{m=0}^{p} q^{m+1} \Phi_{m}(0, \alpha, z ; q)$,

(ii) $\Psi(0, \alpha, z ; q)=(1-q) \sum_{m=0}^{p} q^{m+1} \Phi_{m}(0, \alpha, z ; q)$,

(iii) $\Psi^{\prime}(0, \alpha, z ; q)=\left(z q ; q^{2}\right)_{\infty} \Phi(0, \alpha, z ; q)+\sum_{m=0}^{\infty}\left(z q ; q^{2}\right)_{m+1} q^{2 m+3} \Phi_{m}(0, \alpha, z ; q)$,

(iv) $q^{p+2} \Phi_{p}(0, \alpha, z ; q)=\Psi_{p}(0, \alpha, z ; q)-(1-q) \sum_{m=0}^{p} q^{-(m+2)} \Psi_{m}(0, \alpha, z ; q)$,

(v) $\chi_{p}(0, \alpha, z ; q)=q^{2 p+3}\left\{\mathrm{X}_{p}(0, \alpha, z ; q)-z \chi_{p}(0, \alpha, z ; q)\right\}+\left(1-q^{2}\right) \sum_{m=0}^{p} \begin{gathered}q^{2 m+1}\left\{\mathrm{X}_{m}(0, \alpha, z ; q)\right. \\ \left.-z \chi_{m}(0, \alpha, z ; q)\right\},\end{gathered}$

(vi) $\chi(0, \alpha, z ; q)=\left(1-q^{2}\right) \sum_{m=0}^{p} q^{2 m+1}\left\{\mathrm{X}_{m}(0, \alpha, z ; q)-z \chi_{m}(0, \alpha, z ; q)\right\}$,

we give detailed proof of (i) and for others we indicate only parameters taken.

\section{Proof of (i) \& (ii)}

Taking $\alpha_{r}=\frac{q^{r(r+1) / 2} z^{2 r} \alpha^{r}}{\left(z q ; q^{2}\right)_{r+1}}, \beta_{r}=q^{r+1}$ in Eq.(3), we have;

$$
\begin{aligned}
& \sum_{r=0}^{p} \frac{q^{r(r+1) / 2} z^{2 r} \alpha^{r} q^{r+1}}{\left(z q ; q^{2}\right)_{r+1}}=q^{p+2} \sum_{r=0}^{p} \frac{q^{r(r+1) / 2} z^{2 r} \alpha^{r}}{\left(z q ; q^{2}\right)_{r+1}}+\sum_{m=0}^{p}\left(q^{m+1}-q^{m+2}\right) \sum_{r=0}^{m} \frac{q^{r(r+1) / 2} z^{2 r} \alpha^{r}}{\left(z q ; q^{2}\right)_{r+1}} \\
& \sum_{r=0}^{p} \frac{q^{(r+1)(r+2) / 2} z^{2 r} \alpha^{r}}{\left(z q ; q^{2}\right)_{r+1}}=q^{p+2} \sum_{r=0}^{p} \frac{q^{r(r+1) / 2} z^{2 r} \alpha^{r}}{\left(z q ; q^{2}\right)_{r+1}}+(1-q) \sum_{m=0}^{p} q^{m+1} \sum_{r=0}^{m} \frac{q^{r(r+1) / 2} z^{2 r} \alpha^{r}}{\left(z q ; q^{2}\right)_{r+1}} \\
& \Psi_{p}(0, \alpha, z ; q)=q^{p+2} \Phi_{p}(0, \alpha, z ; q)+(1-q) \sum_{m=0}^{p} q^{m+1} \Phi_{p}(0, \alpha, z ; q) .
\end{aligned}
$$

which gives (i) and letting $p \rightarrow \infty$, we get (ii). 


\section{Proof of (iii)}

Taking $\alpha_{r}=\frac{q^{r(r+1) / 2} z^{2 r} \alpha^{r}}{\left(z q ; q^{2}\right)_{r+1}}, \beta_{r}=\left(z q ; q^{2}\right)_{r+1}$ in Eq. (3) and letting $p \rightarrow \infty$, we get (iii).

\section{Proof of (iv)}

Taking $\alpha_{r}=\frac{q^{(r+1)(r+2) / 2} z^{2 r} \alpha^{r}}{\left(z q ; q^{2}\right) r+1}, \beta_{r}=\frac{1}{q^{r+1}}$ in Eq. (3), we get (iv).

\section{Proof of (v) \& (vi)}

$$
\begin{aligned}
& \mathrm{X}_{m}(0, \alpha, z ; q)=\sum_{r=0}^{m} \frac{(-1)^{r} q^{r^{2}} z^{2 r} \alpha^{r}}{(-z q ; q)_{2 r}} \\
& =\sum_{r=0}^{m} \frac{(-1)^{r} q^{r^{2}} z^{2 r} \alpha^{r}\left(1+z q^{2 r+1}\right)}{(-z q ; q)_{2 r+1}} \\
& =\sum_{r=0}^{m} \frac{(-1)^{r} q^{r^{2}} z^{2 r} \alpha^{r}}{(-z q ; q)_{2 r}}+z \sum_{r=0}^{m} \frac{(-1)^{r} q^{r^{2}+2 r+1} z^{2 r} \alpha^{r}}{(-z q ; q)_{2 r}} \\
& =\sum_{r=0}^{m} \frac{(-1)^{r} q^{r^{2}} z^{2 r} \alpha^{r}}{(-z q ; q)_{2 r+1}}+z \chi_{m}(0, \alpha, z ; q) .
\end{aligned}
$$

Taking $\alpha_{r}=\frac{(-1)^{r} q^{r^{2}} z^{2 r} \alpha^{r}}{\left(-z q ; q^{2}\right)_{2 r+1}}, \beta_{r}=q^{2 r+1}$ in Eq. (3) and using Eq. (6), we get (v) and letting $p \rightarrow$ $\infty$, in (v), we get (vi).

\section{Relation between generalized mock theta functions of tenth order and third order}

First, we define generalized mock theta functions of third order. To avoid confusion, we have put suffix 3 for third order;

$$
\begin{aligned}
& f_{3}(t, \alpha, z ; q)=\frac{1}{(t)_{\infty}} \sum_{n=0}^{\infty} \frac{(t)_{n} q^{n^{2}} z^{2 n} \alpha^{n}}{(-z q ; q)_{n}^{2}}, \quad \varphi_{3}(t, \alpha, z ; q)=\frac{1}{(t)_{\infty}} \sum_{n=0}^{\infty} \frac{(t)_{n} q^{n^{2}} z^{2 n} \alpha^{n}}{\left(-z q^{2} ; q^{2}\right)_{n}}, \\
& \psi_{3}(t, \alpha, z ; q)=\frac{1}{(t)_{\infty}} \sum_{n=0}^{\infty} \frac{(t)_{n} q^{(n+1)^{2}} z^{2 n} \alpha^{n}}{\left(z q ; q^{2}\right)_{n+1}}, \quad v_{3}(t, \alpha, z ; q)=\frac{1}{(t)_{\infty}} \sum_{n=0}^{\infty} \frac{(t)_{n} q^{n^{2}+n} z^{2 n} \alpha^{n}}{\left(-z q ; q^{2}\right)_{n+1}}
\end{aligned}
$$

The following relations are true for generalized mock theta functions of third order and tenth order.

$$
\begin{aligned}
& \text { (i) } \Phi_{\mathrm{p}}\left(0, \alpha, z^{2} ; q^{2}\right)=\frac{z^{2 p}}{\left(-z q ; q^{2}\right)_{p+2}} v_{3 p}(0, \alpha, z ; q)+\sum_{m=0}^{p} \frac{z^{2 m}}{\left(z q ; q^{2}\right)_{m+2}}\left(1-z^{2}-z q^{2 m+3}\right) v_{3 m}(0, \alpha, z ; q), \\
& \text { (ii) } \Phi\left(0, \alpha, 1 ; q^{2}\right)=\frac{1}{\left(q ; q^{2}\right)_{\infty}} v_{3}(0, \alpha, 1 ; q)+\sum_{m=0}^{\infty} \frac{1}{\left(q ; q^{2}\right)_{m+2}}\left(-q^{2 m+3}\right) v_{3 m}(0, \alpha, 1 ; q), \\
& \text { (iii) } \mathrm{X}_{\mathrm{p}}(0, \alpha, z ; q)=\frac{1}{\left(-z q ; q^{2}\right)_{p+1}} \phi_{3 p}(0, \alpha, z ;-q)+\sum_{m=0}^{p} \frac{z q^{2 m+1}}{\left(-z q ; q^{2}\right)_{m+1}} \phi_{3 m}(0, \alpha, z ;-q),
\end{aligned}
$$


(iv) $\mathrm{X}(0, \alpha, z ; q)=\frac{1}{\left(-z q ; q^{2}\right)_{\infty}} \phi_{3}(0, \alpha, z ;-q)+\sum_{m=0}^{\infty} \frac{z q^{2 m+1}}{\left(-z q ; q^{2}\right)_{m+1}} \phi_{3 m}(0, \alpha, z ;-q)$.

\section{Proof of (i) \& (ii)}

Taking $\alpha_{r}=\frac{q^{r(r+1)} z^{2 r} \alpha^{r}}{\left(-z q ; q^{2}\right)_{r+1}}$ and $\beta_{r}=\frac{z^{2 r}}{\left(z q ; q^{2}\right)_{r+1}}$ in (3), we have;

$\sum_{r=0}^{p} \frac{q^{r(r+1)} z^{2 r} \alpha^{r}}{\left(-z q ; q^{2}\right)_{r+1}} \frac{z^{2 r}}{\left(z q ; q^{2}\right)_{r+1}}=\frac{z^{2 p+2}}{\left(z q ; q^{2}\right)_{p+2}} \sum_{r=0}^{p} \frac{q^{r(r+1)} z^{2 r} \alpha^{r}}{\left(-z q ; q^{2}\right)_{r+1}}+\sum_{m=0}^{p}\left(\frac{z^{2 m}}{\left(z q ; q^{2}\right)_{m+1}}-\frac{z^{2 m+2}}{\left(z q ; q^{2}\right)_{m+2}}\right) \sum_{r=0}^{m} \frac{q^{r(r+1)} z^{2 r} \alpha^{r}}{\left(-z q ; q^{2}\right)_{r+1}}$

On simplification we get (i). Putting $z=1$, making $p \rightarrow \infty$ in (i), we get (ii).

\section{Proof of (iii) \& (iv)} (iv).

Taking $\alpha_{r}=\frac{(-1)^{r} q^{r^{2}} z^{2 r} \alpha^{r}}{\left(-z q^{2} ; q^{2}\right)_{r}}$ and $\beta_{r}=\frac{1}{\left(-z q ; q^{2}\right)_{r}}$ in (3), we get (iii) and making $p \rightarrow \infty$ in (iii), we get

\section{Continued fraction representation}

We express generalized tenth order mock theta functions $\mathrm{X}(0, \alpha, z ; q)$ and $\Phi(0, \alpha, z ; q)$ as a continued fraction. For this we require the following 2 lemmas.

Lemma 1 Let following expression be true;

$I(\alpha, z ; q)+\frac{\alpha z^{2} q}{(1+z q)} I(\alpha, z q ; q)-\frac{\alpha z^{3} q^{3}}{(1+z q)\left(1+z q^{2}\right)} I\left(\alpha, z q^{2} ; q\right)=1$.

where, $I(\alpha, z ; q)=\sum_{n=0}^{\infty} \frac{(-1)^{n} q^{n^{2}} z^{2 n} \alpha^{n}}{(-z q ; q)_{2 n}}=\mathrm{X}(0, \alpha, z ; q)$.

\section{Proof of Lemma 1;}

Let

$\mathrm{I}(\alpha, \mathrm{z} ; \mathrm{q})=\sum_{n=0}^{\infty} \frac{(-1)^{n} q^{n^{2}} z^{2 n} \alpha^{n}}{(-z q ; q)_{2 n}}=1+\sum_{n=1}^{\infty} \frac{(-1)^{n} q^{n^{2}} z^{2 n} \alpha^{n}}{(-z q ; q)_{2 n}}$

Replacing $n \rightarrow n+1$, we have;

$$
\begin{aligned}
& \mathrm{I}(\alpha, \mathrm{z} ; \mathrm{q})=1-\sum_{n=0}^{\infty} \frac{(-1)^{n} q^{(n+1)^{2}} z^{2 n+2} \alpha^{n+1}}{(-z q ; q)_{2 n+2}} \\
& =1-\frac{z^{2} \alpha}{(1+z q)} \sum_{n=0}^{\infty} \frac{(-1)^{n} q^{(n+1)^{2}} z^{2 n} \alpha^{n}}{\left(-z q^{2} ; q\right)_{2 n+1}} \\
& =1-\frac{z^{2} \alpha}{(1+z q)} \sum_{n=0}^{\infty} \frac{(-1)^{n} q^{(n+1)^{2}} z^{2 n} \alpha^{n}}{\left(-z q^{2} ; q\right)_{2 n}\left(1+z q^{2 n+2}\right)} \\
& =1-\frac{z^{2} \alpha}{(1+z q)} \sum_{n=0}^{\infty} \frac{(-1)^{n} q^{(n+1)^{2}} z^{2 n} \alpha^{n}}{\left(-z q^{2} ; q\right)_{2 n}} \frac{\left(1+z q^{2 n+2}-z q^{2 n+2}\right)}{\left(1+z q^{2 n+2}\right)}
\end{aligned}
$$




$$
\begin{aligned}
& =1-\frac{z^{2} \alpha}{(1+z q)} \sum_{n=0}^{\infty} \frac{(-1)^{n} q^{(n+1)^{2}} z^{2 n} \alpha^{n}}{\left(-z q^{2} ; q\right)_{2 n}}\left(1-\frac{z q^{2 n+2}}{1+z q^{2 n+2}}\right) \\
& =1-\frac{z^{2} \alpha q}{(1+z q)} \sum_{n=0}^{\infty} \frac{(-1)^{n} q^{n^{2}+2 n} z^{2 n} \alpha^{n}}{\left(-z q^{2} ; q\right)_{2 n}}+\frac{\alpha z^{3} q^{3}}{(1+z q)\left(1+z q^{2}\right)} \sum_{n=0}^{\infty} \frac{(-1)^{n} q^{n^{2}+4 n} z^{2 n} \alpha^{n}}{\left(-z q^{3} ; q\right)_{2 n}} \\
& I(\alpha, z ; q)=1-\frac{\alpha z^{2} q}{(1+z q)} I(\alpha, z q ; q)+\frac{\alpha z^{3} q^{3}}{(1+z q)\left(1+z q^{2}\right)} I\left(\alpha, z q^{2} ; q\right) \\
& I(\alpha, z ; q)+\frac{\alpha z^{2} q}{(1+z q)} I(\alpha, z q ; q)-\frac{\alpha z^{3} q^{3}}{(1+z q)\left(1+z q^{2}\right)} I\left(\alpha, z q^{2} ; q\right)=1
\end{aligned}
$$

which proves the lemma 1 .

Lemma 2 Let $\mathrm{J}(\alpha, z ; q)=\alpha z^{2} q \mathrm{~J}(\alpha q, z ; q)+z q \mathrm{~J}\left(\alpha q^{2}, z ; q\right)+1$ be true where, J $(\alpha, z ; q)=\sum_{n=0}^{\infty} \frac{q^{n(n+1) / 2} z^{2 n} \alpha^{n}}{\left(z q ; q^{2}\right) n+1}=\Phi(0, \alpha, z ; q)$.

\section{Proof of Lemma 2;}

Let $\mathrm{J}(\alpha, z ; q)=\sum_{n=0}^{\infty} \frac{q^{n(n+1) / 2} z^{2 n} \alpha^{n}}{\left(z q ; q^{2}\right)_{n+1}}$

Replacing $\alpha$ by $\alpha q$ and multiplying by $q$ we have;

$\mathrm{qJ}(\alpha q, z ; q)=q \sum_{n=0}^{\infty} \frac{q^{n(n+1) / 2} z^{2 n}(\alpha q)^{n}}{\left(z q ; q^{2}\right)_{n+1}}=\sum_{n=0}^{\infty} \frac{q^{(n+1)(n+2) / 2} z^{2 n} \alpha^{n}}{\left(z q ; q^{2}\right)_{n+1}}$

Making $n \rightarrow(n-1)$, we have;

$$
\begin{aligned}
& q J(\alpha q, z ; q)=\sum_{n=1}^{\infty} \frac{q^{n(n+1) / 2} z^{2 n-2} \alpha^{n-1}}{\left(z q ; q^{2}\right)_{n}} \\
& =\frac{1}{z^{2} \alpha} \sum_{n=1}^{\infty} \frac{q^{\frac{n(n+1)}{2}} z^{2 n} \alpha^{n}\left(1-z q^{2 n+1}\right)}{\left(z q ; q^{2}\right)_{n+1}} \\
& =\frac{1}{z^{2} \alpha} \sum_{n=1}^{\infty} \frac{q^{\frac{n(n+1)}{2}} z^{2 n} \alpha^{n}}{\left(z q ; q^{2}\right)_{n+1}}-\frac{q}{z \alpha} \sum_{n=1}^{\infty} \frac{q^{\frac{n(n+5)}{2}} z^{2 n} \alpha^{n}}{\left(z q ; q^{2}\right)_{n+1}} \\
& =\frac{1}{z^{2} \alpha} \sum_{n=1}^{\infty} \frac{q^{\frac{n(n+1)}{2}} z^{2 n} \alpha^{n}}{\left(z q ; q^{2}\right)_{n+1}}-\frac{q}{z \alpha} \sum_{n=1}^{\infty} \frac{q^{\frac{n(n+5)}{2}} z^{2 n} \alpha^{n}}{\left(z q ; q^{2}\right)_{n+1}}-\frac{1}{z^{2} \alpha(1-z q)}+\frac{q}{z \alpha(1-z q)} \\
& q J(\alpha q, z ; q)=\frac{1}{z^{2} \alpha} J(\alpha, z ; q)-\frac{q z}{z^{2} \alpha} J\left(\alpha q^{2}, z ; q\right)-\frac{1}{z^{2} \alpha} \\
& \mathrm{J}(\alpha, z ; q)=\alpha z^{2} q \mathrm{~J}(\alpha q, z ; q)+z q \mathrm{~J}\left(\alpha q^{2}, z ; q\right)+1 .
\end{aligned}
$$

Now we are ready to give a continued fraction representation of generalized mock theta functions. 
From lemma 1;

$$
\begin{aligned}
& I(\alpha, z ; q)+\frac{\alpha z^{2} q}{(1+z q)} I(\alpha, z q ; q)-\frac{\alpha z^{3} q^{3}}{(1+z q)\left(1+z q^{2}\right)} I\left(\alpha, z q^{2} ; q\right)=1 . \\
& \frac{I(\alpha, z ; q)}{I(\alpha, z q ; q)}=\left(\frac{1}{I(\alpha, z q ; q)}-\frac{\alpha z^{2} q}{(1+z q)}\right)+\frac{\alpha z^{3} q^{3}}{(1+z q)\left(1+z q^{2}\right)} \frac{1}{\frac{I(\alpha, z q ; q)}{I\left(\alpha, z q^{2} ; q\right)}} \\
& \frac{I(\alpha, z ; q)}{I(\alpha, z q ; q)}=M(\alpha, z ; q)+\frac{N(\alpha, z ; q)}{\frac{I(\alpha, z q ; q)}{I\left(\alpha, z q^{2} ; q\right)}}=M(\alpha, z ; q)+\frac{N(\alpha, z ; q)}{M(\alpha, z q ; q)+\frac{N(\alpha, z q ; q)}{M\left(\alpha, z q^{2} ; q\right)+\frac{N\left(\alpha, z q^{2} ; q\right)}{I\left(\alpha, z q^{3} ; q\right)}}}
\end{aligned}
$$

where,$M(\alpha, z ; q)=\left(\frac{1}{I(\alpha, z q ; q)}-\frac{\alpha z^{2} q}{(1+z q)}\right)$ and $N(\alpha, z ; q)=\frac{\alpha z^{3} q^{3}}{(1+z q)\left(1+z q^{2}\right)}$

From lemma 2;

$$
\begin{aligned}
& \mathrm{J}(\alpha, z ; q)=\alpha z^{2} q \mathrm{~J}(\alpha q, z ; q)+z q \mathrm{~J}\left(\alpha q^{2}, z ; q\right)+1 . \\
& \frac{J(\alpha, z ; q)}{J(\alpha q, q ; q)}=\left(\alpha z^{2} q+\frac{1}{J(\alpha q, z ; q)}\right)+\frac{z q}{\frac{J(\alpha q, z ; q)}{J\left(\alpha q^{2}, z ; q\right)}} \\
& \frac{\mathrm{J}(\alpha, z ; q)}{\mathrm{J}(\alpha q, z ; q)}=M(\alpha, z ; q)+\frac{z q}{\frac{J(\alpha q, z ; q)}{I\left(\alpha q^{2}, z ; q\right)}} \\
& =M(\alpha, z ; q)+\frac{z q}{M(\alpha q, z ; q)+\frac{z q}{M\left(\alpha q^{2}, z ; q\right)+\frac{z q}{J\left(\alpha q^{3}, z ; q\right)}}}
\end{aligned}
$$

where, $M(\alpha, z ; q)=\alpha z^{2} q+\frac{1}{J(\alpha q, z ; q)}$

\section{$q$-integral representation of generalized mock theta functions of tenth order}

Thomae and Jackson [7] defined the $q$-integral;

$$
\int_{0}^{1} f(t) d_{q} t=(1-q) \sum_{n=0}^{\infty} f\left(q^{n}\right) q^{n}
$$

using the limiting case of $q$-beta integral we have;

$\frac{1}{\left(q^{x} ; q\right)_{\infty}}=\frac{(1-q)^{-1}}{(q ; q)_{\infty}} \int_{0}^{1} t^{x-1}(t q ; q)_{\infty} d_{q} t$

we now give the integral representation for these generalized tenth order mock theta functions.

Theorem 1 We have following integral representation;

(i) $\Phi\left(q^{t}, \alpha, z ; q\right)=\frac{(1-q)^{-1}}{(q ; q)_{\infty}} \int_{0}^{1} u^{t-1}(u q ; q)_{\infty} \Phi(0, \alpha u, z ; q) d_{q} u$, 
(ii) $\Psi\left(q^{t}, \alpha, z ; q\right)=\frac{(1-q)^{-1}}{(q ; q)_{\infty}} \int_{0}^{1} u^{t-1}(u q ; q)_{\infty} \Psi(0, \alpha u, z ; q) d_{q} u$,

(iii) $\mathrm{X}\left(q^{t}, \alpha, z ; q\right)=\frac{(1-q)^{-1}}{(q ; q)_{\infty}} \int_{0}^{1} u^{t-1}(u q ; q)_{\infty} \mathrm{X}(0, \alpha u, z ; q) d_{q} u$,

(iv) $\chi\left(q^{t}, \alpha, z ; q\right)=\frac{(1-q)^{-1}}{(q ; q)_{\infty}} \int_{0}^{1} u^{t-1}(u q ; q)_{\infty} \chi(0, \alpha u, z ; q) d_{q} u$.

\section{Proof}

We give a detailed proof of (i) only remaining are similar, so omitted.

$\Phi(t, \alpha, z ; q)=\frac{1}{(t)_{\infty}} \sum_{n=0}^{\infty} \frac{(t)_{n} q^{n(n+1) / 2} z^{2 n} \alpha^{n}}{\left(z q ; q^{2}\right)_{n+1}}$

replace $t$ by $q^{t}$ we have;

$\Phi\left(q^{t}, \alpha, z ; q\right)=\frac{1}{\left(q^{t}\right)_{\infty}} \sum_{n=0}^{\infty} \frac{\left(q^{t}\right)_{n} q^{n(n+1) / 2} z^{2 n} \alpha^{n}}{\left(z q ; q^{2}\right)_{n+1}}$

$=\sum_{n=0}^{\infty} \frac{q^{n(n+1) / 2} z^{2 n} \alpha^{n}}{\left(z q ; q^{2}\right)_{n+1}\left(q^{t+n} ; q\right)_{\infty}}$

with the help of Eq. (8), we have;

$\Phi\left(q^{t}, \alpha, z ; q\right)=\frac{(1-q)^{-1}}{(q ; q)_{\infty}} \int_{0}^{1} u^{n+t-1}(u q ; q)_{\infty} \sum_{n=0}^{\infty} \frac{q^{n(n+1) / 2} z^{2 n} \alpha^{n}}{\left(z q ; q^{2}\right)_{n+1}} d_{q} u$,

from definition put $t=0$

$\Phi(0, \alpha, z ; q)=\sum_{n=0}^{\infty} \frac{q^{n(n+1) / 2} z^{2 n} \alpha^{n}}{\left(z q ; q^{2}\right)_{n+1}}$

so, we have;

$\Phi\left(q^{t}, \alpha, z ; q\right)=\frac{(1-q)^{-1}}{(q ; q)_{\infty}} \int_{0}^{1} u^{t-1}(u q ; q)_{\infty} \Phi(0, \alpha u, z ; q) d_{q} u$.

\section{Multibasic expansion}

The following bibasic expansion will be used to give multibasic expansion for the generalized mock theta functions;

\section{Theorem 2}

$\sum_{k=0}^{\infty} \frac{\left(1-a p^{k} q^{k}\right)\left(1-b p^{k} q^{-k}\right)(a, b ; p)_{k}(c, a / b c ; q)_{K} q^{k}}{(1-a)(1-b)(q, a q / b ; q)_{k}(a p / c, b c p ; p)_{k}} \sum_{m=0}^{\infty} \alpha_{m+k}$ 
$=\sum_{m=0}^{\infty} \frac{(a p, b p ; p)_{m}(c q, a q / b c ; q)_{m} q^{m}}{(q, a q / b ; q)_{m}(a p / c, b c p ; p)_{m}} \alpha_{m}$

Using the summation formula [7, (3.6.7), p.71] and [10, Lemma 10, p.57], we have Theorem 2. We will consider following cases of Theorem 2.

Case I Letting $q \rightarrow q^{2}$ and $c \rightarrow \infty$ in Theorem 2, we have;

$\sum_{k=0}^{\infty} \frac{\left(1-a p^{k} q^{2 k}\right)\left(1-b p^{k} q^{-2 k}\right)(a, b ; p)_{k} q^{k^{2}+k}}{(1-a)(1-b)\left(q^{2}, a q^{2} / b ; q^{2}\right)_{k} b^{k} p^{\frac{k^{2}+k}{2}}} \sum_{m=0}^{\infty} \alpha_{m+k}$

$=\sum_{m=0}^{\infty} \frac{(a p, b p ; p)_{m} q^{m^{2}+m}}{\left(q^{2}, a q^{2} / b ; q^{2}\right)_{m} b^{m} p^{\frac{m^{2}+m}{2}}} \alpha_{m}$

Case II. Letting $q \rightarrow q^{3}$ and $c \rightarrow \infty$ in Theorem 2, we have;

$$
\begin{aligned}
& \sum_{k=0}^{\infty} \frac{\left(1-a p^{k} q^{3 k}\right)\left(1-b p^{k} q^{-3 k}\right)(a, b ; p)_{k} q^{\frac{3 k^{2}+3 k}{2}}}{(1-a)(1-b)\left(q^{3}, a q^{3} / b ; q^{3}\right)_{k} b^{k} p^{\frac{k^{2}+k}{2}}} \sum_{m=0}^{\infty} \alpha_{m+k} \\
& =\sum_{m=0}^{\infty} \frac{(a p, b p ; p)_{m} q^{\frac{3 m^{2}+3 m}{2}}}{\left(q^{3}, a q^{3} / b ; q^{3}\right)_{m} b^{m} p^{\frac{m^{2}+m}{2}}} \alpha_{m}
\end{aligned}
$$

Theorem 3 We have following multibasic expansion for generalized tenth order mock theta functions;

(i) $\Phi(t, \alpha, z ; q)=\frac{1}{(t)_{\infty}} \sum_{k=0}^{\infty} \frac{\left(1-t q^{3 k-1}\right)\left(1-q^{-k+1}\right)(t ; q)_{k-1} q^{\frac{k^{2}+k}{2}} z^{2 k} \alpha^{k}}{\left(1-q^{k+1}\right)\left(z q ; q^{2}\right)_{k+1}}$

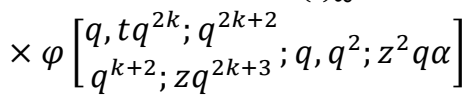

(ii) $\Psi(t, \alpha, z ; q)=\frac{1}{(t)_{\infty}} \sum_{k=0}^{\infty} \frac{\left(1-t q^{3 k-1}\right)\left(1-q^{-k+1}\right)(t ; q)_{k-1} q^{\frac{k^{2}+3 k+2}{2}} z^{2 k} \alpha^{k}}{\left(1-q^{k+1}\right)\left(z q ; q^{2}\right)_{k+1}}$

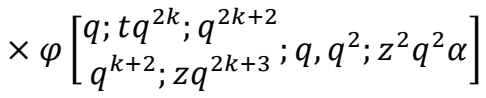

(iii) $\mathrm{X}(t, \alpha, z ; q)=\frac{1}{(t)_{\infty}} \sum_{k=0}^{\infty} \frac{\left(1-t q^{4 k-1}\right)\left(1-q^{-2 k+2}\right)(t ; q)_{k-1}(-1)^{k} q^{k^{2}} z^{2 k} \alpha^{k}}{\left(1-q^{k+2}\right)(-z q ; q)_{2 k}}$

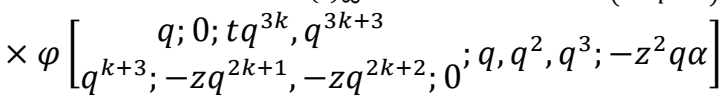

(iv) $\chi(t, \alpha, z ; q)=\frac{1}{(t)_{\infty}} \sum_{k=0}^{\infty} \frac{\left(1-t q^{4 k-1}\right)\left(1-q^{-2 k+2}\right)(t ; q)_{k-1}(-1)^{k} q^{(k+1)^{2} z^{2 k} \alpha^{k}}}{\left(1-q^{k+2}\right)(-z q ; q)_{2 k+1}}$

$\times \varphi\left[\begin{array}{c}q ; 0 ; t q^{3 k}, q^{3 k+3} \\ q^{k+3} ;-z q^{2 k+2},-z q^{2 k+3} ; 0 ; q, q^{2}, q^{3} ;-z^{2} q^{3} \alpha\end{array}\right]$ 
Proof of (i) Let $a=t / q, \quad b=q, p=q, \alpha_{m} \frac{\left(q^{2}, t ; q^{2}\right)_{m} q^{m} z^{2 m} \alpha^{m}}{\left(q^{2} ; q\right)_{m}\left(z q ; q^{2}\right)_{m+1}}$ in (11), we have;

$\sum_{k=0}^{\infty} \frac{\left(1-t q^{3 k-1}\right)\left(1-q^{1-k}\right)(t / q, q ; q)_{k} q^{k^{2}+k}}{(1-t / q)(1-q)\left(q^{2}, t ; q^{2}\right)_{k} b^{k} q^{\frac{k^{2}+k}{2}}} \sum_{m=0}^{\infty} \frac{\left(q^{2}, t ; q^{2}\right)_{m+k} q^{m+k} z^{2 m+2 k} \alpha^{m+k}}{\left(q^{2} ; q\right)_{m+k}\left(z q ; q^{2}\right)_{m+k+1}}$

$=\sum_{m=0}^{\infty} \frac{\left(t, q^{2} ; q\right)_{m} q^{m^{2}+m}}{\left(q^{2}, t ; q^{2}\right)_{m} q^{m} q^{\frac{m^{2}+m}{2}}} \frac{\left(q^{2}, t ; q^{2}\right)_{m} q^{m} z^{2 m} \alpha^{m}}{\left(q^{2} ; q\right)_{m}\left(z q ; q^{2}\right)_{m+1}}$

$\sum_{k=0}^{\infty} \frac{\left(1-t q^{3 k-1}\right)\left(1-q^{1-k}\right)(t / q, q ; q)_{k} q^{\frac{k^{2}+k}{2}} z^{2 k} \alpha^{k}}{(1-t / q)(1-q)\left(q^{2} ; q\right)_{k}\left(z q ; q^{2}\right)_{k+1}} \sum_{m=0}^{\infty} \frac{\left(q^{2 k+2} ; q^{2}\right)_{m}\left(t q^{2 k} ; q^{2}\right)_{m} q^{m} z^{2 m} \alpha^{m}}{\left(q^{k+2} ; q\right)_{m}\left(z q^{2 k+3} ; q^{2}\right)_{m}}$

$=\sum_{m=0}^{\infty} \frac{(t ; q)_{m} q^{\frac{m^{2}+m}{2}} z^{2 m} \alpha^{m}}{\left(z q ; q^{2}\right)_{m+1}}$

$\sum_{k=0}^{\infty} \frac{\left(1-t q^{3 k-1}\right)\left(1-q^{1-k}\right)(t ; q)_{k-1} q^{\frac{k^{2}+k}{2}} z^{2 k} \alpha^{k}}{\left(z q ; q^{2}\right)_{k+1}\left(1-q^{k+1}\right)} \varphi\left[\begin{array}{c}q, t q^{2 k} ; q^{2 k+2} \\ q^{k+2} ; z q^{2 k+3}\end{array} q, q^{2} ; z^{2} q \alpha\right]$

$=(t ; q)_{\infty} \Phi(t, \alpha, z ; q)$

Proof of (ii)

Let $a=t / q, \quad b=q, \quad p=q, \alpha_{m} \frac{\left(q^{2}, t ; q^{2}\right)_{m} q^{2 m+1} z^{2 m} \alpha^{m}}{\left(q^{2} ; q\right)_{m}\left(z q ; q^{2}\right)_{m+1}}$ in

\section{Proof of (iii)}

Let $a=t / q, \quad b=q^{2}, \quad p=q, \alpha_{m} \frac{\left(q^{3}, t ; q^{3}\right)_{m}(-1)^{m} q^{m} z^{2 m} \alpha^{m}}{\left(q^{3} ; q\right)_{m}(-z q ; q)_{2 m}}$ in

\section{Proof of (iv)}

Let $a=t / q, \quad b=q^{2}, \quad p=q, \alpha_{m} \frac{\left(q^{3}, t ; q^{3}\right)_{m}(-1)^{m} q^{3 m+1} z^{2 m} \alpha^{m}}{\left(q^{3} ; q\right)_{m}(-z q ; q)_{2 m+1}}$ in

\section{Special cases and Ramanujan's continued fraction}

From the definition of generalized mock theta functions, we have; 
$\mathrm{X}(t, \alpha, z ; q)=\frac{1}{(t)_{\infty}} \sum_{n=0}^{\infty} \frac{(t)_{n}(-1)^{n} q^{n^{2}} z^{2 n} \alpha^{n}}{(-z q ; q)_{2 n}}$

$\chi(t, \alpha, z ; q)=\frac{1}{(t)_{\infty}} \sum_{n=0}^{\infty} \frac{(t)_{n}(-1)^{n} q^{(n+1)^{2}} z^{2 n} \alpha^{n}}{(-z q ; q)_{2 n+1}}$

Taking $t=0, \alpha=-1$ and $z=-1$ in (13) and (14), we have;

$\mathrm{X}(0,-1,-1 ; q)=\sum_{n=0}^{\infty} \frac{q^{n^{2}}}{(q ; q)_{2 n}}$

$\chi(0,-1,-1 ; q)=q \sum_{n=0}^{\infty} \frac{q^{n^{2}+2 n}}{(q ; q)_{2 n+1}}$

From [14, eqn. (A.158), p.186];

$\frac{G\left(q^{4}\right)}{\chi(-q)}=\frac{f\left(-q^{8},-q^{12}\right)}{\psi(-q)}=\sum_{n=0}^{\infty} \frac{q^{n^{2}}}{(q ; q)_{2 n}}$

From [14, eqn. (A.157), p.185];

$\frac{H\left(q^{4}\right)}{\chi(-q)}=\frac{f\left(-q^{4},-q^{16}\right)}{\psi(-q)}=\sum_{n=0}^{\infty} \frac{q^{n^{2}+2 n}}{(q ; q)_{2 n+1}}$

From Eqs. (15) and (17), we have;

$\mathrm{X}(0,-1,-1 ; q)=\frac{G\left(q^{4}\right)}{\chi(-q)}$

From Eqs. (16) and (18), we have;

$\chi(0,-1,-1 ; q)=\frac{H\left(q^{4}\right)}{\chi(-q)}$

where $G(q)$ and $H(q)$ are Ramanujan functions and are numerator and denominator of celebrated Ramanujan's continued fraction $R(q)$. 
Dividing Eqs. (19) by (20), we get;

$q \frac{\mathrm{X}(0,-1,-1 ; q)}{\chi(0,-1,-1 ; q)}$

$=R\left(q^{4}\right)$

\section{Applications of mock theta functions}

Mock theta functions appear in the theory of umbral moonshine. Atish et al. [11] showed that mock modular forms are related to the degeneracies of quantum black holes in $\mathrm{N}=4$ string theories.

\section{Conclusions}

The advantage of generalization is that by specializing the parameters we can connect the mock theta functions with Ramanujan's continued fractions. So, the results obtained for mock theta functions reduced to continued fractions of Ramanujan. As mock theta functions are called mysterious functions. I think, these relations between mock theta functions and continued fractions will help in the study of mock theta functions.

\section{Acknowledgement}

I am thankful to Prof. Bhaskar Srivastava for his guidance.

\section{References}

[1] GE Andrews. Mock theta functions. Proc. Sympos. Pure Math. 1989; 49, 283-98.

[2] GE Andrews and AJ Yee. Some identities associated with mock theta functions $\omega(\mathrm{q})$ and $v(\mathrm{q})$. Ramanujan J. 2019; 48, 613-22.

[3] BC Berndt, A Dixit and R Gupta. Generalizations of the Andrews-Yee identities associated with the mock theta functions $\omega(\mathrm{q})$ and $v(\mathrm{q})$, Available at: https://arxiv.org/abs/2101.11779, accessed January 2021.

[4] S Bhargava. The Rogers-Ramanujan identities. Srinivasa Ramanujan (1887 - 1920). Mac-Millan India, Madras, India, 1988, p. 75-81.

[5] S Bhargava and C Adiga. On some continued fraction identities of Srinivasa Ramanujan. Proc. Amer. Math. Soc. 1984; 92, 13-8.

[6] YS Choi. Tenth order mock theta functions in Ramanujan's lost notebook. Invent. Math. 1999; 136, 497-569.

[7] YS Choi. Tenth order mock theta functions in 'Ramanujan's lost notebook II. Adv. Math. 2000; 156, 180-285.

[8] YS Choi. Tenth order mock theta functions in 'Ramanujan's lost Notebook IV. Trans. Amer. Math. Soc. 2002; 354, 705-33.

[9] YS Choi. Tenth order mock theta functions in 'Ramanujan's lost notebook III. Proc. London Math. Soc. 2007; 94, 26-52.

[10] YS Choi. The basic bilateral hypergeometric series and the mock theta functions. Ramanujan J. 2011; 24, 345-86

[11] A Dabholkar, S Murthy and D Zagier. Quantum black holes, wall crossing, and mock modular forms, Available at: https://arxiv.org/abs/1208.4074, accessed August 2020.

[12] RY Denis. On certain q-series and continued fractions. Math. Student. 1983; 44,70-6.

[13] RY Denis. On basic hypergeometric functions and continued fractions. Math. Student. 1984; 52, 12936.

[14] RY Denis. On certain summation of q-series and identities of Rogers-Ramanujan type. J. Math. Phys. Sci. 1988; 22, 87-99. 
[15] G Gasper and M Rahman. Basic hypergeometric series. Cambridge University Press, Cambridge 1990.

[16] FH Jackson. Basic integration. Quart. J. Math. 1951; 2, 1-16.

[17] ED Rainville. Special function. Chelsea Publishing Company, New York, 1960.

[18] S Ramanujan. Collected paper. Cambridge University Press, Cambridge, 1927.

[19] AV Sills. An invitation to the Rogers-Ramanujan identities. CRC Press, Boca Raton, Florida, 2017.

[20] B Srivastava. A study of Fq-functions connected with Ramanujan's tenth order mock theta functions. Math. J. Okayama Univ. 2004; 46, 131-9.

[21] B Srivastava. Ramanujan's mock theta functions. Math. J. Okayama Univ. 2005; 47, 163-74.

[22] GN Watson. The final problem: An account of the mock theta functions. J. London Math. Soc. 1936; 11, 55-80.

[23] S Zwegers. The tenth-order mock theta functions revisited. Bull. London Math. Soc. 2010; 42, 30111. 